\title{
Correlation of medial/lateral rotation of the humerus with glenohumeral translation
}

\author{
Thomas P Branch, Orlando Avilla, Lisa London, William C Hutton
}

\begin{abstract}
Objectives-To correlate glenohumeral translation in the anterior/posterior direction with medial and lateral rotation of the humerus. In addition, the length of the anterior and posterior component of the glenohumeral capsuloligamentous complex was varied in order to gain insight into the contribution of each component to limiting translation. All measurements were made with the humerus positioned at $90^{\circ}$ of abduction and $0^{\circ}$ of flexion/ extension.
\end{abstract}

Methods-Six fresh cadaveric shoulders were used. Each scapula was mounted in a cement pot to rest it in its correct anatomical position. Seven tests were carried out on each shoulder. A series of measurements of translation of the humerus in the anterior direction and posterior direction were taken at $20^{\circ}$ intervals of lateral rotation and then at $20^{\circ}$ intervals of medial rotation until the limit of lateral or medial rotation had clearly been reached (test 1). The capsuloligamentous complex was then incised and a beaded chain and catches were sutured across the joint to mimic the capsuloligamentous complex at different lengths (tests 2 to 7 ).

Results/Conclusions-(a) When the glenohumeral capsuloligamentous complex is intact, the humerus translates maximally in the glenoid (between 20 and $30 \mathrm{~mm}$ ) when the humerus is between $40^{\circ}$ and $100^{\circ}$ of lateral rotation. (b) As the glenohumeral capsuloligamentous complex increases in length, so does the extent of translation. (c) In medial rotation, the length of the posterior capsule, rather than the length of the anterior capsule, has the greater effect on anterior/posterior translation. (d) In lateral rotation the length of the anterior capsule, rather than the length of the posterior capsule, has the greater effect on anterior/posterior translation. (e) The glenohumeral ligamentous complex acts more as a cuff, enclosing the joint, rather than as a sling, as is commonly thought.

(Br F Sports Med 1999;33:347-351)

Keywords: humerus; glenohumeral translation; rotation; ligaments; shoulder

The glenohumeral joint is the most mobile joint in the body and the glenohumeral capsuloligamentous complex is the main provider of stability to the joint. The glenohumeral capsuloligamentous complex consists of an anterior and a posterior component. The anterior component is comprised of the anterior inferior, middle, and superior glenohumeral ligaments, while the posterior component is comprised of the posterior inferior glenohumeral ligament and the rest of the posterior capsule. ${ }^{1-17}$ The capsuloligamentous complex is thought to provide a moving sling which stabilises and limits glenohumeral motion. ${ }^{5}$

Damage to the glenohumeral ligaments of the shoulder as the result of trauma is rarely caused by a direct translatory blow to the humerus; most often the applied force has some component of rotation. ${ }^{510} 1618$ The combination of forces to produce abduction, extension, and lateral rotation applied to the arm may result in an anterior dislocation. ${ }^{5} 1113151819$ Axial loading of the abducted medially rotated arm may produce a posterior dislocation..$^{511} 121518$ The mechanism of these injuries involves rotation yet, historically, the measurement of the injury has generally been stated only in terms of translation (never rotation)..$^{5}{ }^{12-17}{ }^{20-22}$ It was therefore decided to try to correlate translation of the humerus with medial and lateral rotation of the humerus, with respect to the glenoid.

It was decided at the outset to limit the measurements to the humerus positioned at $90^{\circ}$ of abduction and $0^{\circ}$ of flexion/extension. This position is defined as the humerus directly perpendicular to the plane of the glenoid. In addition to correlating glenohumeral translation with medial/lateral rotation, it was also decided to vary the length of the anterior/ inferior and posterior/inferior component of the glenohumeral capsuloligamentous complex in order to gain insight into each component's contribution to limiting translation.

\section{Materials and methods}

CADAVERIC MATERIAL

Six fresh (unembalmed) cadaveric shoulders (plus humeri) were used (three right and three left). The shoulders were from two men and two women, mean age 63 (range 55-72) years. After the shoulders had been thawed, the musculature was completely dissected away, leaving only the bony and ligamentous structures of the humerus and scapula (including coracoid and acromion). At this stage the shoulders were examined to ensure that there was no degeneration and that the glenohumeral capsuloligamentous complex was completely intact and undamaged; two other specimens (not included in our experiments) were discarded at this stage. 


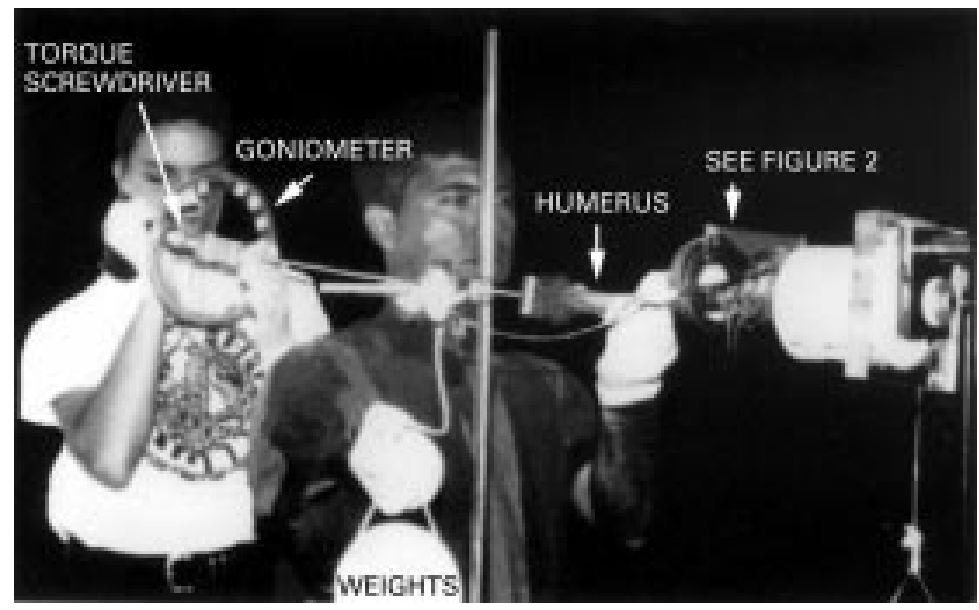

Figure 1 The experimental set up used to measure translation of the internally/externally rotated humerus. Reproduced with permission of the copyright holder.

SPECIMEN MOUNTING

After dissection, the scapula was "potted". This consisted of inserting the medial border of the scapula into the open end of a PVC pipe (10 $\mathrm{cm}$ diameter by $10 \mathrm{~cm}$ long), and then filling the pipe with dental cement. The potted scapula and humerus were then mounted on to a vertical stand. The glenohumeral joint was thus able to rest in the correct anatomical position to simulate the standing position. The scapula was mounted so that the plane of the glenoid was perpendicular to the ground and the acromion was rotated $23^{\circ}$ from the horizontal in the plane of the glenoid (fig 1).

\section{APPLICATION OF THE BEADED CHAIN}

Into the medial side of the glenohumeral capsuloligamentous complex (the side of the ligament at the attachment to the glenoid) were sutured segments of "beaded chain" (the type commonly used to attach pens to desks or drain plugs to sinks; fig 2). Adjacent beads on the chain were $4.2 \mathrm{~mm}$ apart when the chain was taut. An ample free length of chain was provided to allow subsequent attachment to "chain catches" on the scapular side of the ligament. The catches were screwed into the bony humerus. Five chain and catch sets, located at the $2,5,6,7$, and 10 o'clock positions were used. Thus when the ligament was cut (see below) the chain (and catch sets)

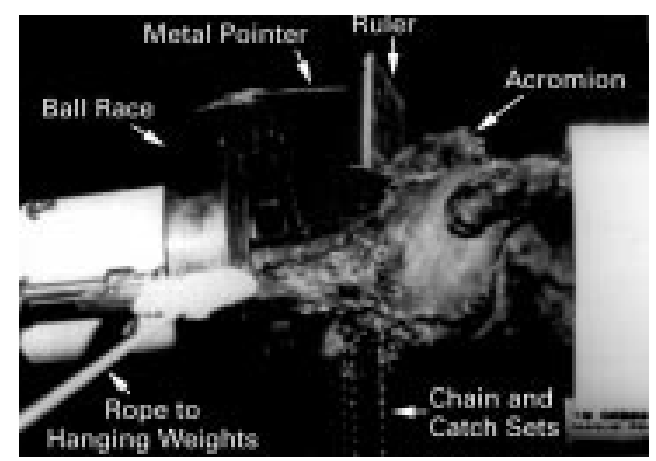

Figure 2 The scapula is shown potted in cement in a length of PVC pipe. The chain and catches used to mimic the components of the capsuloligamentous complex at different lengths are also shown. Reproduced with permission of the copyright holder. could be used to mimic the normal length of the components of the ligamentous complex. Each length of chain could also be increased by increments of $4.2 \mathrm{~mm}$. Anterior and posterior lengths could be adjusted independently of each other, allowing multiple permutations of simulated capsular ligaments at different lengths. This chain and catch method has been described previously. ${ }^{23}$

\section{METHOD OF MEASURING ANTERIOR/POSTERIOR} TRANSLATION

The following procedure was carried out to measure the extent of anterior or posterior translation of the humerus with respect to the acromion. A ball race, $10 \mathrm{~cm}$ internal diameter, was secured around the shaft of the proximal humerus. A small metal rod $10 \mathrm{~mm}$ in length and $0.5 \mathrm{~mm}$ in diameter was attached so as to project axially out from the outer circumference of the ball race. This metal rod was lined up with a ruler attached to the acromion (fig 2). The extent of translation was determined by a single observer standing in the same position (fig 1) for each measurement while another researcher held the humerus in the required position. Repeated observations were made, involving at least four measurements, until a single consistent reading was obtained and recorded. The ball race allowed the metal rod to be rotated back to its original position to permit comparable readings to be made when the humerus had been rotated medially or laterally.

All measurements of translation were made with the distal end of the humerus placed in a position of $90^{\circ}$ of abduction and $0^{\circ}$ of flexion/ extension. No attempt was made to compress the humeral head in the glenoid fossa while the transitional measurements were being made so as to ensure that the capsuloligamentous complex was allowed to reach its own mechanical equilibrium. Before measurement, two equal and opposite forces were applied to counteract sag of the humeral head, while allowing the ligaments to maintain the humeral head in equilibrium within the glenoid. These forces of $15 \mathrm{~N}$ of anterior directed force and $15 \mathrm{~N}$ of posterior force were applied through weights, low-friction pulleys, and rope attached at the circumference of the ball race. Anterior and posterior translation were produced by the application of an additional force of 26.7 $\mathrm{N}$ - that is, $6 \mathrm{lb}$-in the required direction.

ROTATION OF THE HUMERUS

Medial and lateral rotation of the humerus with respect to the scapula was defined using an imaginary line passing through the medial and lateral epicondyles. The humerus was defined as being in $0^{\circ}$ of medial/lateral rotation when this line was perpendicular to the horizontal. In the present experiment this "neutral" position is defined with reference to the scapula and not to the thorax as it would be in a clinical study.

To measure medial and lateral rotation, a threaded rod $1.5 \mathrm{~cm}$ in diameter and $100 \mathrm{~cm}$ long was inserted for half its length up the humerus through a hole drilled in the distal end of the bone, and a "goniometer" was 
Table 1 Mean results for the normal shoulders (test 1) and the shoulders with the capsuloligamentous complex lengthened by different amounts (tests 3, 4, and 5). Medial rotation is negative and lateral rotation is positive

\begin{tabular}{lccll}
\hline Humeral rotation & $\begin{array}{l}\text { Normal length } \\
\text { (test 1) }\end{array}$ & $\begin{array}{l}\text { Normal }+4.2 \mathrm{~mm} \\
\text { (test 3) }\end{array}$ & $\begin{array}{l}\text { Normal }+ \\
12.6 \mathrm{~mm} \text { (test 4) }\end{array}$ & $\begin{array}{l}\text { Normal }+ \\
21.0 \mathrm{~mm} \text { (test 5) }\end{array}$ \\
\hline Anterior translation $(\mathrm{mm})$ & & & \\
$-20^{\circ}$ & 3.2 & 6.9 & 11.5 & 15.2 \\
Neutral & 3.7 & 11.5 & 13.1 & 15.8 \\
$20^{\circ}$ & 5.3 & 11.8 & 14.5 & 18.5 \\
$40^{\circ}$ & 12.3 & 14.2 & 17.3 & 19.7 \\
$60^{\circ}$ & 11.3 & 15.2 & 17.5 & 20.8 \\
$80^{\circ}$ & 12.2 & 13.2 & 15.9 & 21.3 \\
$100^{\circ}$ & 10.5 & 12.1 & 14.3 & 22.8 \\
$120^{\circ}$ & 4.5 & 7.1 & 10.2 & 18.7 \\
Posterior translation & $(\mathrm{mm})$ & & & \\
$-20^{\circ}$ & -2.8 & -6.8 & -15.8 & -17.7 \\
Neutral & -6.5 & -10.3 & -19.7 & -22.5 \\
$20^{\circ}$ & -9.3 & -12.7 & -20.1 & -22.5 \\
$40^{\circ}$ & -14.8 & -15.9 & -19.2 & -22.7 \\
$60^{\circ}$ & -13.7 & -15.1 & -16.3 & -20.7 \\
$80^{\circ}$ & -11.5 & -13.8 & -14.9 & -16.3 \\
$100^{\circ}$ & -8.7 & -11.2 & -12.1 & -13.2 \\
$120^{\circ}$ & -4.7 & -8.8 & -9.5 & -10.1 \\
\hline
\end{tabular}

attached firmly to the distal end of the rod (fig $1)$. The goniometer consisted of a $360^{\circ}$ protractor $(20 \mathrm{~cm}$ diameter) with a length of surgical tubing $(1 \mathrm{~cm}$ diameter) glued around the inner edge of its outer diameter. A steel ball ( $3 \mathrm{~mm}$ diameter) was placed inside the plastic tubing so that it could roll freely to its lowest gravitational position. The datum positionthat is, the low point - of the ball was noted and the shoulder was then rotated to the required positions.

Measurements of anterior and posterior translation were made with the humerus unrotated and in medial and lateral rotation. Measurements were made at intervals of $20^{\circ}$ until the limits of medial and lateral rotation were reached. The anatomical maximum of medial and lateral rotation was defined as the rotation for an applied torque of 0.9 N.m as measured with a torque screwdriver (SK Hand Tool Corporation, Chicago, Illinois, USA). This value was chosen after preliminary studies carried out by the authors on their own shoulders.

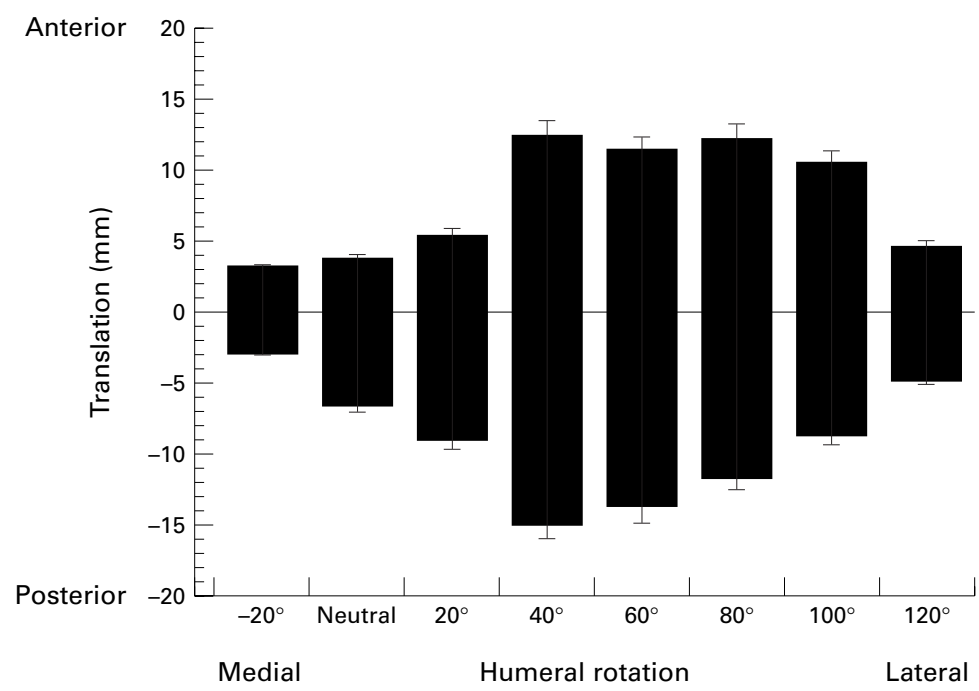

Figure 3 Results for the normal shoulders (test 1). Translation in the anterior and posterior directions is shown against internal/external rotation of the humerus. Results are mean (SEM).
TESTS

This whole procedure of measuring the anterior and posterior translation of the humerus at $20^{\circ}$ intervals up to the anatomical maximum of medial and lateral rotation was carried out first for the normal intact shoulder (test 1). The anterior and posterior portions of the capsuloligamentous complex were then excised from the 12.30 o'clock position to the 11.30 o'clock position, leaving only a $1 \mathrm{~cm}$ bridge at the 12 o'clock position. The chains on the glenoid side were then attached to their corresponding catches on the humeral side, and translation was again measured (test 2). Simulated equal lengthening of the anterior and posterior elements of the capsule was then produced using the beaded chain at different lengths (tests 3, 4, and 5). Simulated posterior and inferior lengthening of the capsuloligamentous complex was effected with the anterior portion at normal length (test 6). Test 7 simulated anterior/inferior lengthening with the posterior part of the complex at normal length. Table 1 gives the lengths of the chains and the angles of rotation for the different tests.

STATISTICAL ANALYSIS

One way and two way analysis of variance was used to determine significance within tests and between tests (Epistat 4.2; Epistat, Richardson, Texas, USA).

\section{Results}

Table 1 and fig 3 give the mean results for the normal (intact) shoulders (test 1 ). (Remember: the humerus was kept at $90^{\circ}$ of abduction and $0^{\circ}$ of flexion/extension.) Translation in the anterior and posterior directions are shown against medial/lateral rotation of the humerus. Translation was at a maximum when the shoulder was laterally rotated between $40^{\circ}$ and $100^{\circ}$. Within this range the overall translation of the humerus in the glenoid (from anterior to posterior) was between $20 \mathrm{~mm}$ and $30 \mathrm{~mm}$. At the extremes of medial/lateral rotation, translation was significantly less than translation at other positions of medial/lateral rotation $(p<0.001$ by single factor analysis of variance).

There was no significant difference $(\mathrm{p}>0.05)$ between the results for the normal intact shoulder (test 1) and the results for the shoulder after the capsuloligamentous complex had been cut but retained at the original length (test 2).

Figure 4 shows the results for the tests in which the chain and catch sets were increased in length by equal amounts at every position (tests 3, 4, and 5) compared with normal. The results show that translation increased as the ligament length increased $(\mathrm{p}<0.001$ by single factor analysis of variance), and medial/lateral rotation increased as the ligament length increased $(p<0.001$ by single factor analysis of variance).

When comparing the effect of selected anterior or posterior capsuloligamentous complex lengthening on anterior and posterior translation, there was a significant $(\mathrm{p}<0.05)$ difference between lengthening the anterior capsule and lengthening the posterior capsule (tests 6 and 


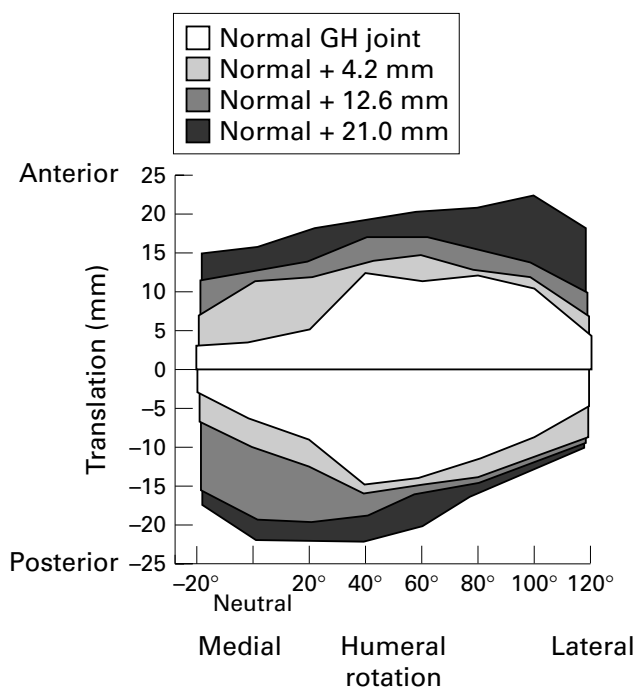

Figure 4 The results for translation are shown against internal/external rotation. The results for the normal

shoulders (test 1) are compared with the results obtained when the chain and catch sets were increased in length by equal amounts at every position (tests 3, 4, and 5). GH, glenohumeral.

7). Lengthening the posterior capsule resulted in a significantly increased anterior/posterior translation only when the joint was near the extreme of medial rotation $\left(20^{\circ}\right.$ of medial rotation) but not when the joint was near the extreme of lateral rotation $\left(120^{\circ}\right.$ of lateral rotation). Similarly, lengthening the anterior capsule resulted in a significantly increased anterior and posterior translation only when the joint was near the extreme of lateral rotation $\left(120^{\circ}\right.$ of lateral rotation) but not when the joint was near the extreme of medial rotation $\left(20^{\circ}\right.$ of medial rotation) (fig 5 ).

The maximum rotation in both directions for three of the six shoulders was found to be

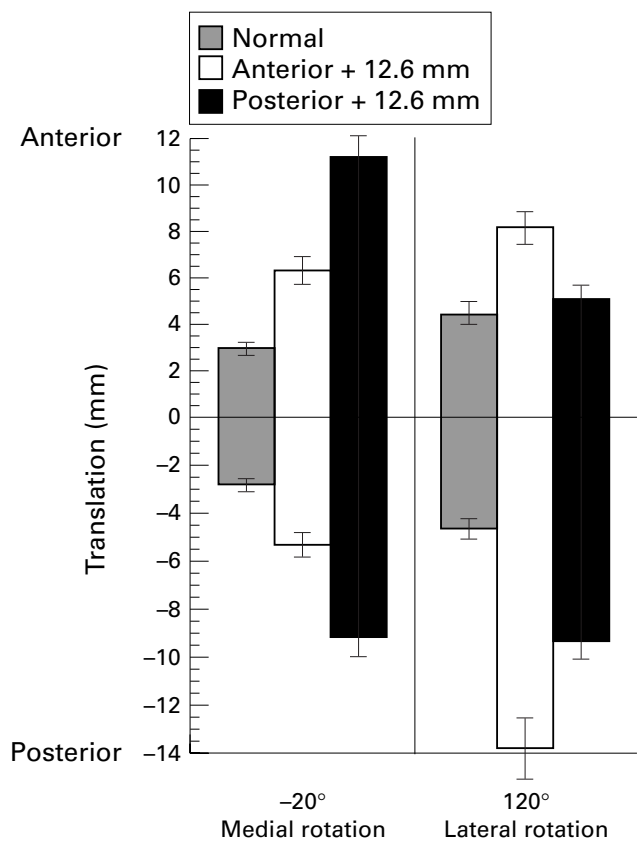

Figure 5 The effect of lengthening the anterior and posterior capsule on anterior and posterior translation at the maxima of internal rotation $\left(20^{\circ}\right.$ of internal rotation) and external rotation $\left(120^{\circ}\right.$ of external rotation) (tests 6 and 7). Results are means (SEM). $20^{\circ}$ of medial and $120^{\circ}$ of lateral. The other three shoulders were able to be rotated nearly $20^{\circ}$ more in both directions. However, for the sake of consistency in fig $5,20^{\circ}$ of medial rotation and $120^{\circ}$ of lateral rotation were used as the limit for our measurements.

\section{Discussion}

Using fig 3, some detail of the overall structure of the glenohumeral capsuloligamentous complex can be interpreted. The ligament appears to be most lax (in terms of allowing the most translation) at about $50^{\circ}$ of lateral rotation. The anatomical range of medial/lateral rotation is about $\pm 70^{\circ}$ from this position-that is, from $120^{\circ}$ of lateral rotation to $20^{\circ}$ of internal rotation), although it was about $\pm 90^{\circ}$ in half of the shoulders. When it rotates, either laterally or medially, from this $50^{\circ}$ position of lateral rotation, the cross fibres of the capsule become tight and so resist anterior and posterior translation at the limits of lateral and medial rotation. This description implies that the ligament acts more as a cuff, enclosing the joint, rather than a sling (as is commonly thought). ${ }^{5}$

The fact that translation increases as the glenohumeral ligamentous complex increases in length is not surprising. Clearly, the longer the ligament, the more loose the cuff, and the greater the ability to translate. The tightening effect, as the humerus laterally and medially rotates in the glenoid, is also indicated on fig 4 by the reduction in translation at the limits of rotation even when the ligament is lengthened by only $4.2 \mathrm{~mm}$. However, when the ligament is lengthened by $12.6 \mathrm{~mm}$ and by $21.0 \mathrm{~mm}$, the reduction in translation at the limits of "normal" rotation is not as apparent. This is an important point in that it indicates that any shoulder surgery that leaves the glenohumeral capsuloligamentous complex more than about $5 \mathrm{~mm}$ longer than normal, risks introducing instability by removing the tightening effect that stabilises the shoulder joint against translation at the limits of normal medial/lateral rotation. A further point is that the limits of normal rotation would also increase if the ligament was longer.

In medial rotation, the posterior capsule had the greater influence on translation, whereas in lateral rotation the anterior capsule had the greater influence on translation (fig 5). When a patient with an unstable shoulder is examined, symptoms are typically elicited when the arm is stressed in lateral or medial rotation. In a previous study of shoulder kinematics, we determined a clinical test for isolating either the anterior or the posterior capsule during rotation. ${ }^{23}$ The anterior capsule can be tested with the arm at $30-40^{\circ}$ of abduction and $0-10^{\circ}$ of flexion/extension; the humerus is then rotated laterally. Similarly, the posterior capsule can be tested with the arm in $60-70^{\circ}$ of abduction and $20-30^{\circ}$ of flexion; the humerus is then rotated medially. The study presented here suggests an alternative clinical test in that the anterior capsule can be independently stressed by translating the humerus anteriorly/ posteriorly while at $90^{\circ}$ abduction, $0^{\circ}$ flexion/ extension, and $120^{\circ}$ lateral rotation. The 
posterior capsule can be independently stressed by translating the humerus anteriorly/ posteriorly while at $90^{\circ}$ of abduction, $0^{\circ}$ flexion/extension, and $20^{\circ}$ medial rotation. It follows that, if symptoms are reproduced by translation while in lateral rotation, the focus of repair should be on the anterior capsule. Similarly, if symptoms are reproduced by translation while in medial rotation, the focus of repair should be on the posterior capsule.

The results presented here are relevant to dislocation of the abducted shoulder joint. When the abducted shoulder is laterally rotated at the time of the dislocation, the anterior glenohumoral capsuloligamentous complex is more likely to be damaged. On the other hand, when the abducted shoulder is medially rotated at the time of dislocation, the posterior glenohumeral capsuloligamentous complex is more likely to be damaged.

1 Bigliani LU, Pollock RG, Soslowsky LJ, et al. Tensile properties of the inferior glenohumeral ligament. $\mathcal{F}$ Orthop Res 1992;10:187-97.

2 Bowen MK, Warren RF. Ligamentous control of shoulder stability based on selective cutting and static translation experiments. Clin Sports Med 1991;10:757-82.

3 Cain PR, Mutschler TA, Fu FH, et al. Anterior stability of the glenohumeral joint. A dynamic model. Am $\mathcal{F}$ Sports Med 1987; 15:144-8.

4 Cooper DE, O'Brien SJ, Warren RF. Supporting layers of the glenohumeral joint. An anatomic study. Clin Orthop the glenohumeral

5 Matsen FA, Fu FH, Hawkins RJ. The shoulder: a balance of mobility and stability. Rosemont, IL: American Academy of Orthopaedic Surgeons, 1993

6 McKernan DJ, Mutschler TA, Rudert MJ, et al. Significance of a partial and full bankart lesion: a biomechanical comparison. Proceedings of the 35th Annual Meeting of the Orthopedic Research Society, 1989:231.

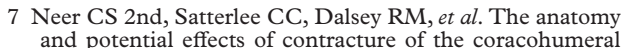
and potential effects of contracture of

8 O'Brien SJ, Neves MC, Aronczky SP, et al. The anatomy and histology of the inferior glenohumeral ligament complex of the shoulder. Am F Sports Med 1990;18:449-56.

9 O'Connell PW, Nuber GW, Mileski RA, et al. The contribution of the glenohumeral ligaments to anterior stability of the shoulder joint. Am $\mathcal{F}$ Sports Med 1990;18:579-84.

10 Ovesen J, Nielsen S. Anterior and posterior shoulder instability. A cadaver study. Acta Orthop Scand 1986;57:324-7.

11 Ovesen J, Nielsen S. Stability of the shoulder joint: cadaver study of stabilizing structures. Acta Orthop Scand 1985;56: 149-51.

12 Schwartz E, Warren RF, O'Brien SJ, et al. Posterior shoulder instability. Orthop Clin North Am 1987;18:409-19.

13 Schwartz RE, O'Brien SJ, Warren RF, et al. Capsular restraints to anterior-posterior motion of the shouder. Orthopaedic Transactions 1988;12:727.

14 Silliman JF, Hawkins RJ. Classification and physical diagnosis of instability of the shoulder. Clin Orthop 1993;291:719.

15 Terry GC, Hammon D, France P, et al. The stabilizing function of passive shoulder restraints. Am 7 Sports Med 1991;19:26-34.

16 Turkel SJ, Panio MW, Marshall JL, et al. Stabilizing mechanisms preventing anterior dislocation of the glenohumeral nisms preventing anterior dislocation of the glen

17 Warner JJ, Deng XH, Warren RF, et al. Static capsuloligamentous restraints to superior-inferior translation of the mentous restraints to superior-inferi

18 Gohlke F, Daum P, Bushe C. The stabilizing function of the glenohumeral joint capsule. Current aspects of the biomechanics of instability. Z Orthop Ihre Grenzgeb 1994;132: $112-19$

19 Moseley HF, Overgaard B. The anterior capsular mechanism in recurrent anterior dislocation of the shoulder. $\mathcal{F}$ Bone foint Surg [Br] 1962;44:913-27.

20 Harryman DT II, Sidles JA, Harris SL, et al. The role of the rotator interval capsule in passive motion and stability of the shoulder. F Bone foint Surg [Am] 1992;74:53-66.

21 Jobe FW, Pink M. Classification and treatment of shoulder dysfunction in the overhead athlete. F Orthop Sports Phys Ther 1993;18:427-32.

22 Kvitne RS, Jobe FW. The diagnosis and treatment of anterior instability in the throwing athlete. Clin Orthop 1993;291:107-23.

23 Branch TP, Lawton RL, Iobst CA, et al. The role of glenohumeral capsular ligaments in internal and external rotation of the humerus. Am F Sports Med 1995;23:632-7.

Take home message

The glenohumeral ligamentous complex acts more as a cuff than a sling as is commonly thought. 